\title{
MODELAGEM DO FENÔMENO DA DETONAÇÃO APLICADA AO MOTOR DE COMBUSTÃO FLEX
}

\author{
André Vinicius Oliveira Maggio ${ }^{1}$, Angelo Maggio Neto ${ }^{1}$, Armando Antônio Maria \\ Laganá ${ }^{1}$, Joao Francisco Justo Filho ${ }^{1}$, Marcos Henrique Carvalho Silva ${ }^{1,2}$, Marco \\ Isola Naufal ${ }^{2}$ \\ ${ }^{1}$ Escola Politécnica de Universidade de São Paulo (EPUSP) \\ ${ }^{2}$ Instituto de Pesquisas Tecnológicas (IPT) - Laboratório de Motores \\ E-mails: andremaggio@usp.br, andremaggio@usp.br, \\ armandolagana@terra.com.br, justo@lme.usp.br, marcoshencarsil@gmail.com, \\ mnaufal@ipt.br
}

\section{RESUMO}

O fenômeno de combustão, conhecido como detonação (knock), causa danos ao motor de combustão interna e, portanto, deve ser evitado. A unidade de controle do motor ECU (Engine Control Unit), ao detectar indícios que a detonação ocorreu durante a combustão, atua sobre o ângulo de ignição de forma a atrasá-lo, evitando que a detonação ocorra. Trabalha-se, portanto, em um ângulo de ignição diferente do ótimo (maximum brake torque timing), chamado de ângulo de limiar, o IKT (incipient knock timing), acarretando perdas de potência. A detonação não é um fenômeno completamente determinístico, uma vez que a própria pressão dentro do cilindro varia de ciclo para ciclo. Desse modo, o foco de se modelar a detonação é prever a intensidade e a probabilidade de sua ocorrência dada a condição de operação e do combustível utilizado pelo motor, pois, cada tipo de combustível tem uma dada condição limite a partir da qual a detonação ocorre. Neste artigo é feito um estudo da probabilidade de ocorrência da detonação e a sua intensidade em função da condição de operação do motor, feito através da análise do sinal do sensor.

\section{INTRODUÇÃO}

O motor de combustão interna produz energia mecânica a partir do trabalho exercido pelos gases quentes sobre o pistão no ciclo de expansão. Nos motores de ciclo Otto, estes gases são produtos da combustão que se inicia a partir da centelha produzida pela vela. Em uma combustão ideal, a centelha produzida pela vela de ignição gera uma frente de chama que percorre a câmara de combustão de forma suave, liberando energia, elevando a temperatura e a pressão dentro do cilindro [1] [2].

A combustão também pode ocorrer a partir de uma ignição espontânea que ocorre sem a necessidade da centelha. Esse processo nessas condições denomina-se 
autoignição da mistura ar-combustível. A autoignição ocorre quando se supera a energia de ativação da reação de combustão, devido as condições de temperatura e pressão dentro do cilindro.

Sob certas condições de operação do motor, a propagação da frente de chama pode provocar uma autoignição na região dos gases que ainda não foram queimados devido a elevação das condições de pressão e de temperatura nesta região conforme visto na Figura 1 [1] [2].

Figura 1 - Fenômeno da detonação dentro do cilindro.

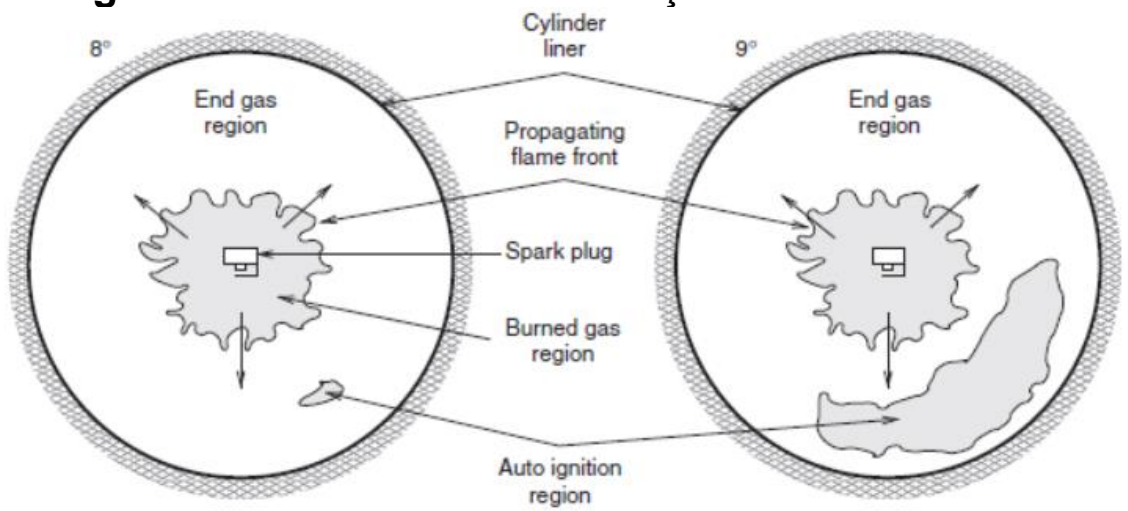

Fonte: [1].

A autoignição ocorrida dentro da região que ainda não sofreu a queima, gera uma segunda frente de chama. Porém, devido às condições de pressão e temperatura dentro da câmara, esta segunda frente de chama se propaga de forma instantânea, provocando uma onda de choque [2] [3]. Este fenômeno é chamado de detonação ou knock, também sendo conhecida como batida de pino devido ao seu som característico. Devido ao aumento de pressão dentro da câmara, provocado pela onda de choque e também devido à colisão entre as duas frentes de chama podem ocorrer outras autoignições [1] [2] [4].

A onda de choque se propaga pelo cilindro colidindo com as suas paredes sendo, parte da sua energia transmitida para a massa do bloco do motor e a energia excedente é refletida novamente no interior do cilindro, formando uma câmara de ressonância. Esta câmara de ressonância faz com que a vibração gerada pela detonação possua uma faixa de frequência característica. Existem formulações empíricas que relacionam a frequência principal em função da geometria do cilindro e da velocidade de propagação do som interna do cilindro, conforme as equações (1) e (2) [1] [5].

$$
\begin{gathered}
v_{\text {som }}=\sqrt{\gamma \cdot R \cdot T} \approx 900 \mathrm{~m} / \mathrm{s} \\
f_{\text {knock }}=\frac{v_{\text {som }} \cdot 1,84}{\pi \cdot D}
\end{gathered}
$$


$f_{\text {knock }}$ : frequência característica da detonação em $\mathrm{Hz}$;

$v_{\text {som }}$ : velocidade de propagação do som dento do cilindro em $\mathrm{m} / \mathrm{s}$;

$D$ : $\quad$ diâmetro do pistão em $\mathrm{m}$;

$R$ : $\quad$ constante dos gases para o ar em J/kgK;

$\gamma: \quad$ razão dos calores específicos, ${ }^{{ }^{\mathrm{p}}} / \mathrm{c}_{\mathrm{v}}$ (adimensional);

$T: \quad$ temperatura dentro do cilindro no momento da detonação em $\mathrm{K}$.

Para o caso do motor Flex, tem-se que cada tipo de combustível tem uma dada condição limite a partir da qual a autoignição ocorrerá. Esta capacidade do combustível resistir a autoignição por compressão é medida pelo índice de octanagem [2] [4]. Historicamente este índice possui essa denominação em função das moléculas da gasolina utilizadas para determinar a escala, o isooctano (alta resistência a detonação) e o n-heptano (baixa resistência a detonação) [4]. Posteriormente, essa propriedade foi estudada em outros combustíveis como o etanol. O primeiro método desenvolvido para mensurar a resistência a autoignição foi o Research Octane Number (RON) que utiliza um motor monocilindro com relação de compressão variável onde são realizados testes simulando o motor a plena carga em uma rotação constante com um determinado combustível ou mistura de combustível. Os resultados obtidos são comparados com misturas de isooctano e $\mathrm{n}$-heptano com octanagem previamente conhecidas. Esse método é descrito em detalhes pela norma ASTM D2699. O segundo método desenvolvido denomina-se Motor Octane Number (MON) que recorre a um motor monocilindro com relação de compressão variável com controle do ponto de ignição, onde são executados testes simulando o motor a plena carga em rotação constante de um combustível ou mistura de combustíveis previamente aquecidos a uma temperatura especifica. Os resultados do teste são comparados a misturas de isooctano e $n$-heptano com octanagem previamente conhecida. A norma ASTM D2700 descreve com mais detalhes o método MON. Existe o índice antidetonante (IAD) que consiste na média aritmética dos valores de octanagem RON e MON.

\section{SISTEMA DE CONTROLE DA DETONAÇÃO}

A detonação, dependendo da intensidade e frequência com que ocorre, pode causar danos ao motor e, portanto, deve ser evitada. Existe um valor do ângulo de ignição que produz um maior toque de saída do motor. Este ângulo ótimo é encontrado na literatura como MBT (maximum brake torque timing). A unidade de controle do motor (ECU - engine control unit) busca o ângulo mais próximo possível do MBT e onde não ocorra a detonação, chamado de ângulo de limiar, o IKT (incipient knock timing). A ECU atua sobre o ponto de ignição, atrasando-o indo em direção ao IKT e, consequentemente, reduzindo a pressão dentro do cilindro, conforme pode-se observar na Figura 2, evitando que a detonação ocorra. Porém, isto também acarreta perdas no rendimento do motor [1] [5]. 
Figura 2 - Pressão no cilindro para diferentes ângulos de ignição.

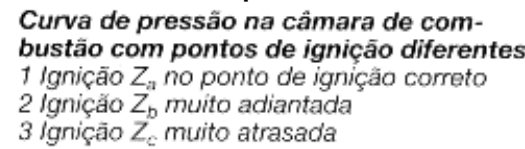

3 Igniçâa $Z$ musto atrasada

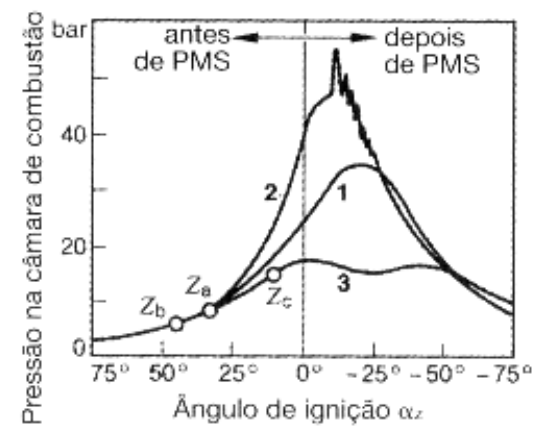

Fonte: [6].

A ECU detecta quando a detonação ocorre através de um sensor especifico instalado junto à superfície do bloco do motor, Figura 3. O sensor de detonação, geralmente, é do tipo piezoelétrico contando com uma massa acoplada a uma cerâmica piezoelétrica que, ao ser submetida a ação de uma aceleração externa, exerce uma força de compressão ou tração na cerâmica devido à inércia gerando uma tensão elétrica na saída do sensor conforme a Figura 3 [6].

Figura 3 - Sensor de detonação (a), e sua curva de resposta característica (b)

$$
\left(1 \mathrm{~g}=9,8 \mathrm{~m} / \mathrm{s}^{2} \mathrm{~g}=9,8 \mathrm{~m} / \mathrm{s}^{2}\right) .
$$

a

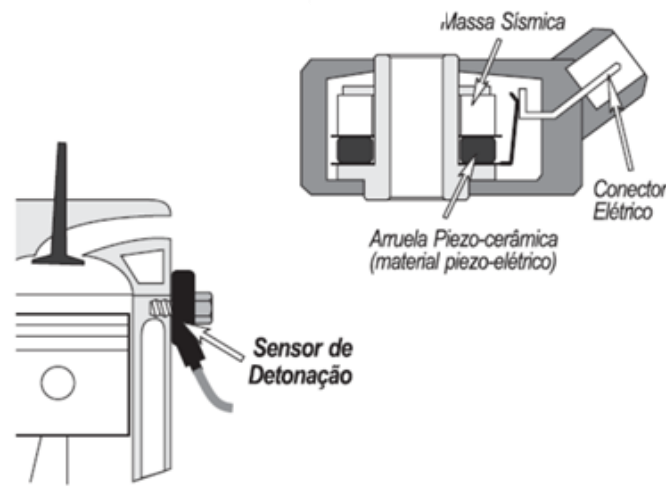

b

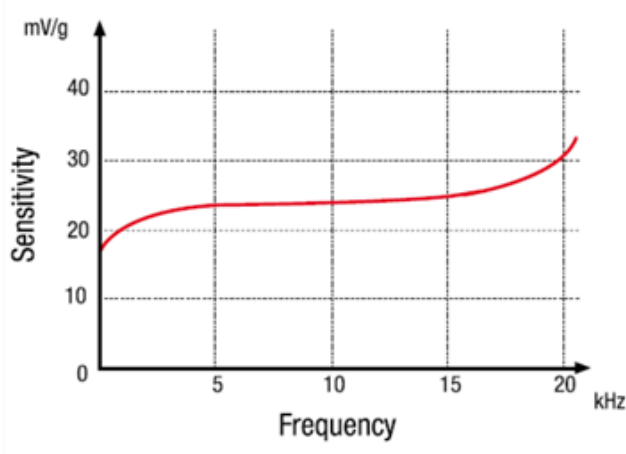

Fonte: a [mte-thomson manual técnico], b [FAE manual técnico].

O sensor de detonação também capta as demais vibrações geradas pelo funcionamento do motor, conforme é possível observar em sua curva característica representada pela Figura 3. Conforme já mencionado, a vibração gerada pela detonação possui uma dada frequência específica que pode ser identificada através da filtragem do sinal do sensor. A detonação não é um fenômeno completamente determinístico, uma vez que a própria pressão dentro do cilindro varia de ciclo para ciclo. Desse modo, o foco em se modelar a detonação é prever a intensidade e a 
probabilidade de sua ocorrência dada a condição de operação do motor [2] [7]. Permitindo que se possa reproduzir o sinal captado pelo sensor.

A probabilidade da detonação ocorrer cresce na medida em que a pressão de pico dentro do cilindro aumenta até o ponto em que a detonação ocorra em todos os ciclos [3]. Porém, o cálculo da pressão de pico dentro do cilindro é não trivial e está além da modelagem exposta neste trabalho. Sugere-se, a partir da análise das referências [3], [7] e [8], levantar a probabilidade de ocorrência da detonação através da análise do sinal do sensor de detonação dadas a condição de operação do motor, como regime de carga, rotação, e composição do combustível utilizado. É adotado como regime de carga a pressão no coletor de admissão.

\section{MODELAGEM DA DETONAÇÃO}

A modelagem tratada neste trabalho visa a reprodução do sinal do sensor em um formato hábil de ser interpretado pela ECU. Para a análise do sinal obtido com o sensor de detonação, utilizou-se de dados disponíveis obtidos a partir de uma vela instrumentada, capaz de medir a pressão interna ao cilindro.

Figura 4 - Resposta característica do sensor de detonação. Gasolina E27, avanço de $30^{\circ} \mathrm{APMS}$, 3500RPM e $90 \mathrm{kPa}$.
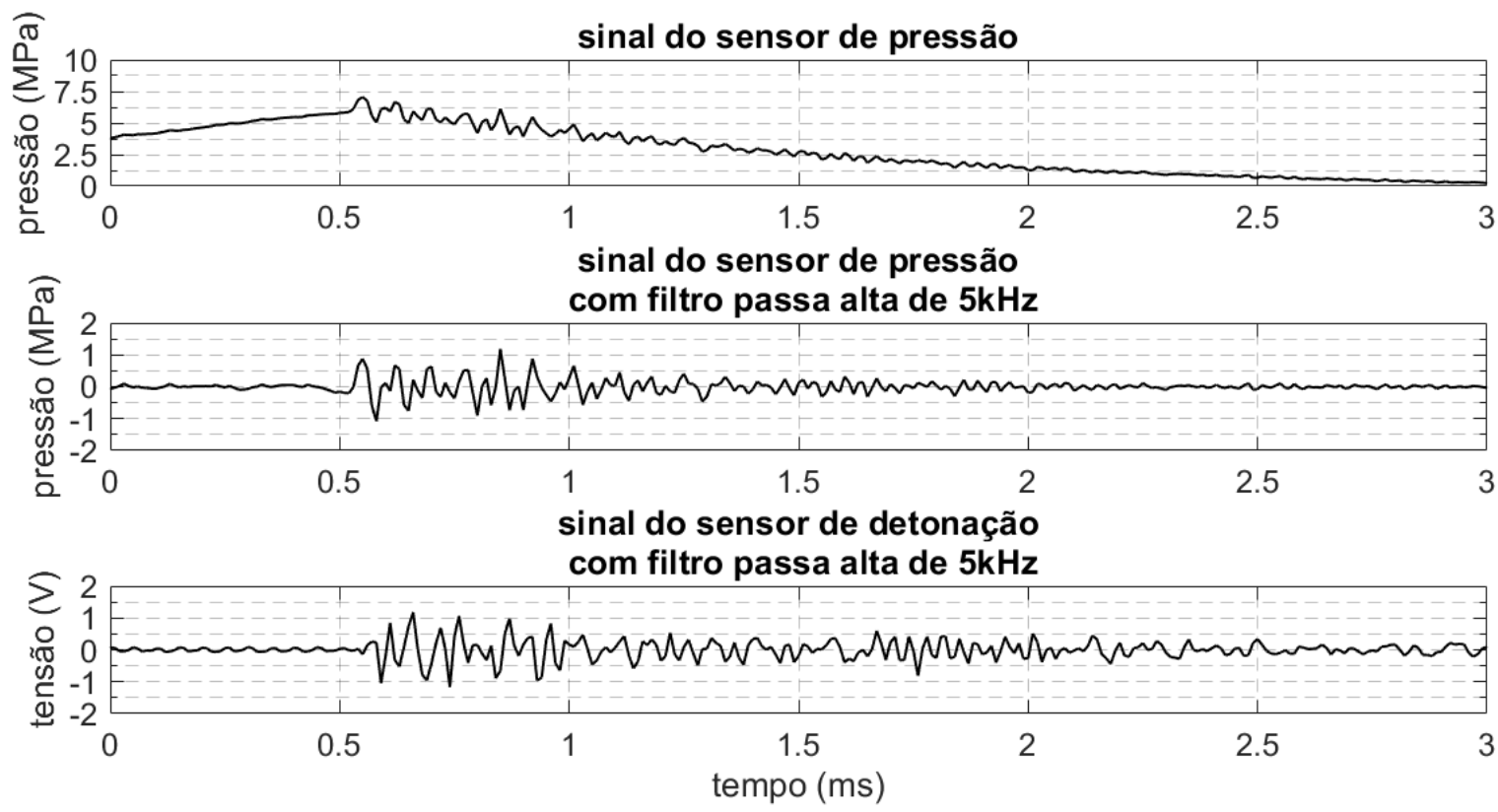

Na Figura 4 tem-se a resposta do sensor de detonação, para uma dada condição em que ocorre a detonação, capitada através das oscilações de pressão medidas pelo sinal do sensor de pressão. Foi feita então a filtragem tanto do sinal de pressão do 
sensor quanto do sinal do sensor de detonação por um filtro passa alta com frequência de corte igual a $5 \mathrm{kHz}$. Faz-se esta etapa de filtragem, pois a vibração característica da detonação é de alta frequência [5]. Para o caso do motor estudado a frequência característica obtida com a equação empírica (2) é de $6,89 \mathrm{kHz}$. Como se dispõe do sinal de pressão, foi possível calcular o ganho do sinal do sensor de pressão com relação ao sinal do sensor de detonação, conforme a equação (3) [9].

$$
G_{\text {sensPres }}=\frac{\left.p_{r m s}\right|_{t=0,6 m s} ^{t=1 m s}-\left.p_{r m s}\right|_{t=2,5 m s} ^{t=3 m s}}{\left.V_{r m s}\right|_{t=0,6 m s} ^{t=1 m s}-\left.V_{r m s}\right|_{t=2,5 m s} ^{t=3 m s}}=0,959 \mathrm{MPa} / V
$$

$G_{\text {sensPres }}:$ ganho do sensor de pressão em $\mathrm{MPa} / \mathrm{V}$;

$p_{r m s}$ valor eficaz do sinal filtrado do sensor de pressão numa dada janela de tempo em MPa;

$V_{r m s}$ valor eficaz do sinal filtrado do sensor de pressão numa dada janela de tempo em V.

Foram feitos ensaios em dinamômetro com o uso de um gerador de função e uma ECU programável. O gerador de função foi utilizado com objetivo de gerara estímulos aplicados ao ângulo de ignição que pudessem ser feitos com relativa precisão e repetibilidade conforme feito em [9]. Primeiramente foi feita a análise espectral do sinal do sensor de detonação utilizando a transformada rápida de Fourier (FFT) [9].

Figura 5 - Espectro do sinal do sensor de detonação para diferentes ângulos de ignição.

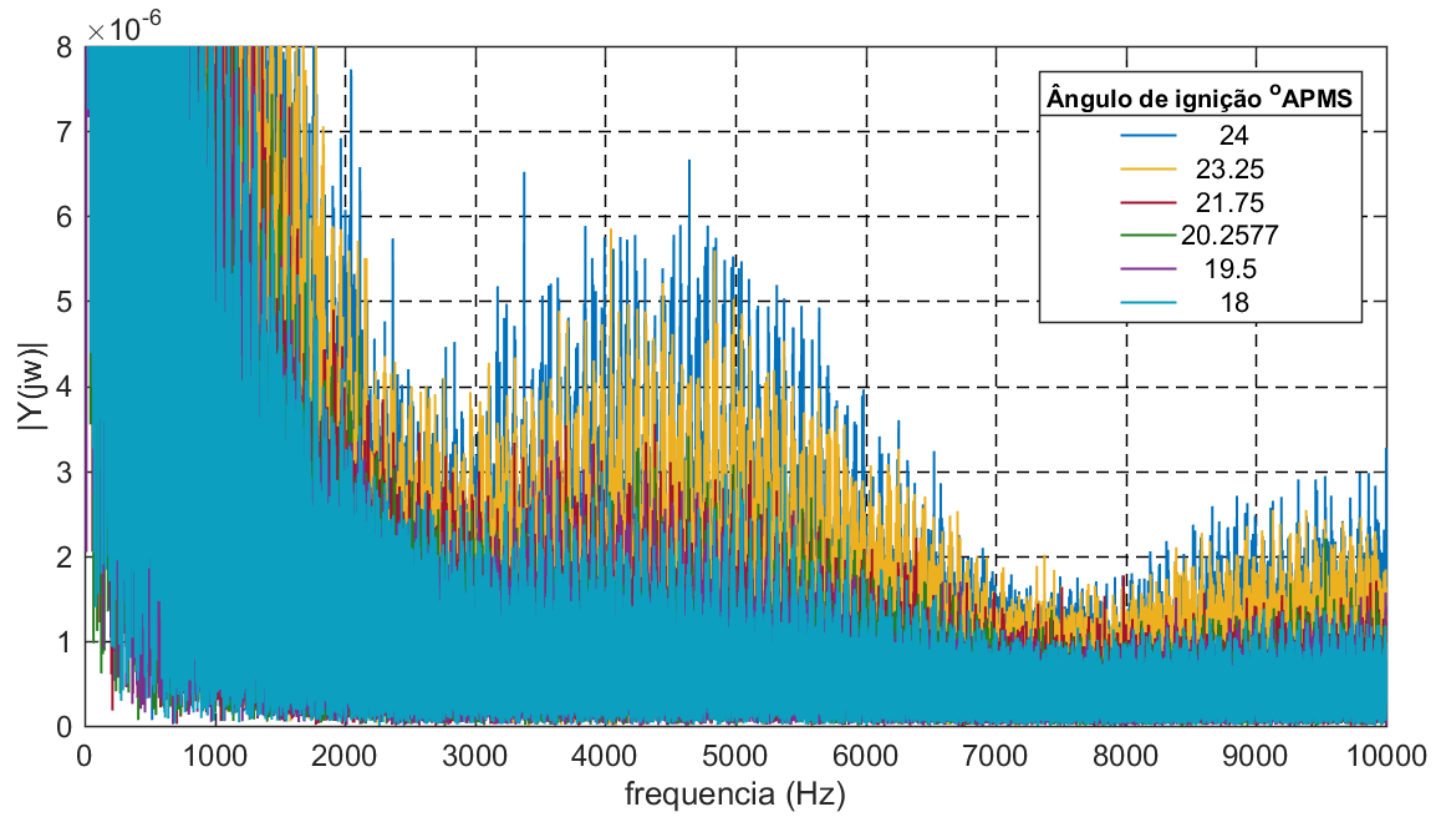

Na Figura 5 é possível observar, através da análise espectral, que conforme se aumenta o valor do ângulo de ignição (avanço de ignição), para uma dada condição 
constante de operação do motor tem-se um aumento da amplitude do espectro nas diferentes faixas de frequência com o aumento do avanço de ignição. $O$ ângulo de ignição é comumente expressado na unidade ${ }^{\circ} \mathrm{APMS}$ ou graus antes do ponto morto superior (PMS). $\mathrm{O}$ aumento da intensidade do espectro ocorre em diversas faixas de frequência além da frequência prevista pela equação empírica (2). A partir da análise espectral foi escolhido um filtro passa-faixa com banda passante de 6,5 a $8,5 \mathrm{kHz}$, para condicionar o sinal obtido com o sensor de detonação, esta faixa de frequência também engloba a frequência obtida pela fórmula empírica, uma vez que a ECU utiliza esta frequência como referência.

A metodologia usada para o processamento do sinal do sensor de detonação consiste em a partir do sinal condicionado pelo filtro passa-faixa descrito, extrair o valor máximo de tensão medida em uma determinada janela de amostras (janela). Esta metodologia se baseia no uso de uma métrica chamada de MAPO (maximum amplitude of the pressure oscillations) que consiste em extrair o valor de pico do sinal filtrado numa dada janela de tempo, conforme equação (4). Usa-se uma janela de 180 graus calculado conforme equação (5), pois a combustão ocorre apenas a cada meia volta do virabrequim. Esta janela percorre o sinal já filtrado e amostrado do sensor de detonação, coletando o valor máximo de sua intensidade.

$$
\begin{gathered}
M A P O=\left.\max (|V(n)|)\right|_{L} \\
L=\frac{\theta \cdot F S}{6 \cdot \overline{\omega_{e}}}
\end{gathered}
$$

$V(n)$ : sinal do sensor de detonação já filtrado e amostrado em V.

Fs: frequência de amostragem em $\mathrm{Hz}$.

$L$ : número de amostras da janela (adimensional);

$\theta$ : janela analisada $\mathrm{em}^{\circ}$ );

$\omega_{e}$ : rotação do motor em RPM.

Para analisar a influência das condições de operação do motor, foram testadas diversas condições possíveis durante os ensaios, variando-se: a rotação do motor, a pressão na admissão (carga do motor), a composição do combustível e o fator lambda. Para cada condição de operação do motor testada durante os ensaios, foi aplicada a metodologia de processamento descrita armazenando-se a média dos valores das diversas tensões máximas e a respectiva variância, a fim de investigar o a influência da condição de operação do motor. 
Figura 6 - Influencias da rotação (a), fator lambda(b), pressão na admissão (c) e temperatura do motor (d) no valor máximo de tensão do sensor de detonação.
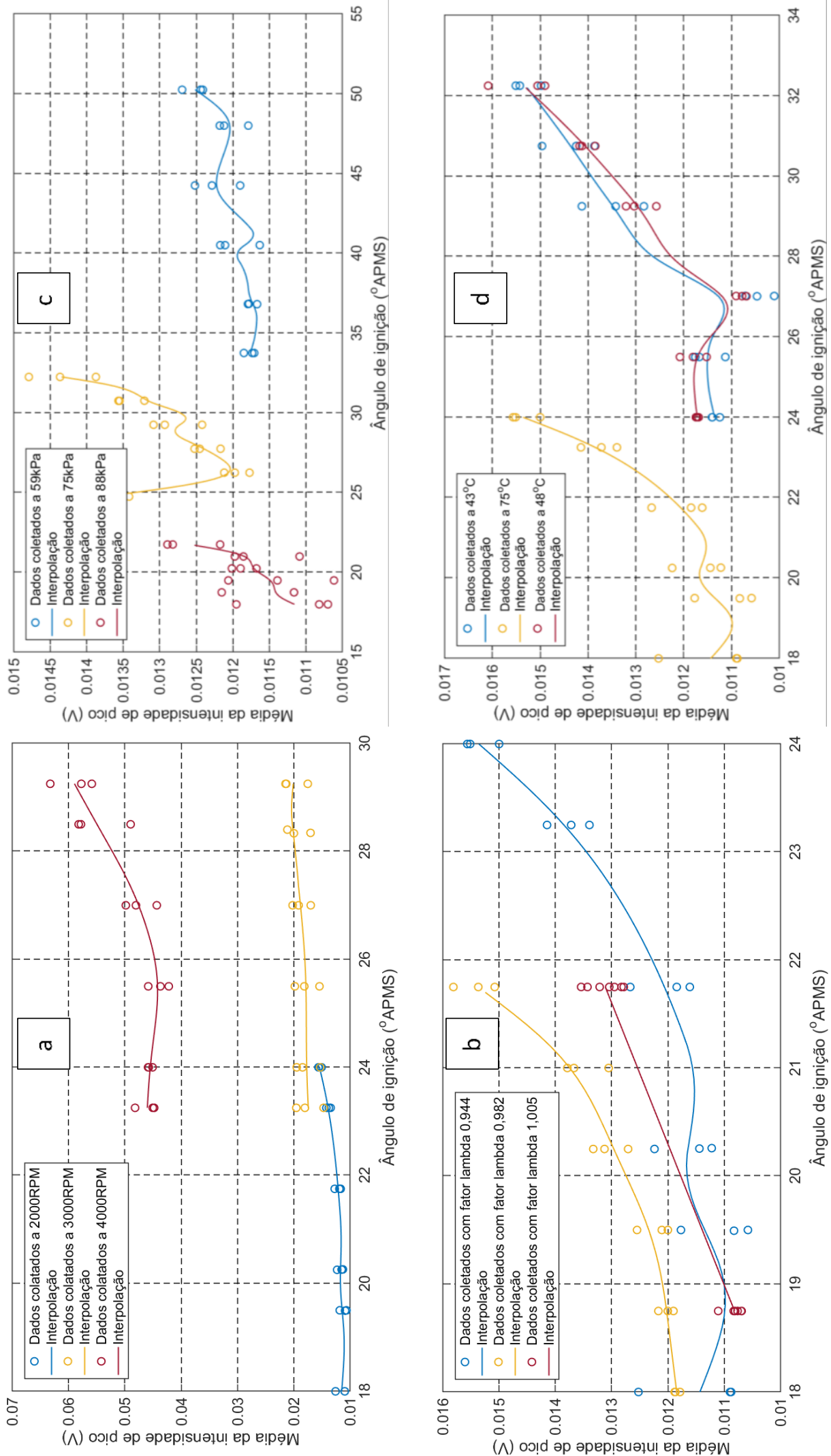
Notou-se, na análise dos resultados experimentais, que tanto a rotação do motor, pressão na admissão, composição do combustível, relação lambda e temperatura do motor impactam significativamente no sinal do sensor de detonação. Na Figura 6a, observa-se o aumento da intensidade de pico médio em função do aumento da rotação do motor enquanto as demais condições permanecem fixas. Há um perceptível aumento não linear na magnitude do pico quando o motor opera em condições de alta rotação (4000RPM). Na Figura 6b, nota-se que a mistura ligeiramente rica (fator lambda menor que 1) provoca maior intensidade de pico médio. Salienta-se que a influência do fator lambda é muito mais modesta que as demais variáveis. Na Figura 6c, nota-se o impacto da pressão no coletor de admissão no sinal do sensor de detonação. Percebeu-se que, para baixas condições de pressão, a amplitude do pico é atenuada. Esse comportamento era esperado, pois isto também diminui a pressão interna ao cilindro. Na Figura $6 \mathrm{~d}$, infere-se a influência da temperatura do motor com o motor aquecido a $75^{\circ} \mathrm{C}$, obtém-se a mesma amplitude de pico em comparação ao motor frio (em torno de $45^{\circ} \mathrm{C}$ ). Considerando o motor se observa que a curva é deslocada, indicando uma menor sensibilidade ao valor de ângulo de ignição quando comparada a curva obtida com o motor aquecido.

Para simular o sinal do sensor de detonação, é necessário reproduzir a amplitude do sinal conforme disposto na seção. Tal sinal é modelado como sendo um pulso senoidal amortecido de forme semelhante ao observado na Figura 4. A partir dos resultados experimentais obtidos utilizou-se uma rede neural para predizer o valor médio de magnitude de pico e sua variância. Estes valores foram obtidos a partir de ensaios feitos para diversas condições de rotação do motor, pressão na admissão, composição do combustível e fator lambda. A saída da rede neural são os valores da amplitude de pico média e de sua variância. Estes valores são utilizados para gerar um número aleatório a partir da distribuição normal (gaussiana), ao se realizar um pulso de disparo. Tal pulso é gerado periodicamente a cada 180 graus. Obtevese o resultado apresentado na Figura 7.

Figura 7 - Simulação do sinal do sensor de detonação.

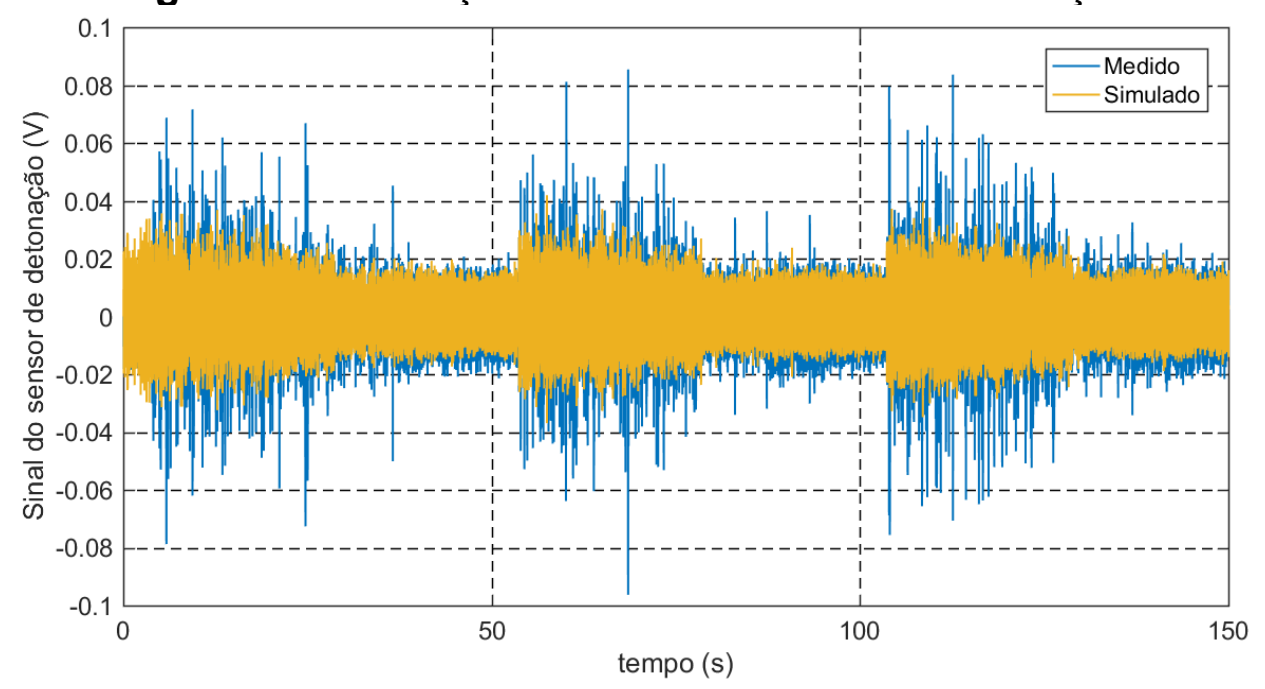




\section{CONCLUSÃO}

No presente trabalho foi apresentada a modelagem do sinal do sensor de estatística do sensor detonação piezoelétrico. Apresentando gráficos referente a análises acerca da influência dos parâmetros descritos. A modelagem tratada, diferente dos modelos determinísticos, pode recorrer tanto a sensores piezoelétricos quanto a transdutores de pressão, possibilitando maior flexibilidade de implementação. Os resultados são satisfatórios e, apesar de em certos intervalos a amplitude de pico do sinal simulado ser inferior ao sinal real, os dois sinais apresentaram mesma intensidade média de pico, que permite ao sistema de controle predizer a ocorrência da detonação.

\section{REFERÊNCIAS}

[1] Eriksson, L.; Nielsen, L. Modeling and control of engines and drivelines. John Wiley \& Sons, 2014.

[2] HEYWOOD, J. B. Internal combustion engine fundamentals. McGraw-Hill c1988. McGraw-Hill, Series in mechanical engineering.

[3] PANZANI, G.; ÖSTMAN, F.; ONDER, C. H. Engine knock margin estimation using in-cylinder pressure measurements. IEEE/ASME Transactions on Mechatronics, v. 22, n. 1, p. 301-311, 2017. DOI: 10.1109/TMECH.2016.2604920

[4] BRUNETTI, F. Motores de combustão interna. São Paulo: Blucher, v. 1. 554 p., 2012.

[5] GUZZELLA, L.; ONDER, C. Introduction to modeling and control of internal combustion engine systems. ed. 2. Springer Science \& Business Media, 2010.

[6] BOSCH, R. Manual de tecnologia automotiva. São Paulo: Blücher ed. 25, 1232 p., 2005.

[7] Steurs, K. F. H. M. Cycle-resolved analysis and modeling of knock in a homogeneous charge spark ignition engine fueled by ethanol and iso-octane., 2014. Tese de Doutorado. ETH Zurich.

[8] XIANG, L.; SONG, E.; DING, Y. A Two-Zone Combustion Model for Knocking Prediction of Marine Natural Gas SI Engines. Energies, v. 11, n. 3, p. 561, 2018.

[9] MAGGIO, A. V. O. Simulação hardware in the loop de um motor de combustão Flex. São Paulo. Dissertação de Mestrado - Escola Politécnica da Universidade de São Paulo. 2019. 